\title{
Simulation of Viscous and Reactive Hypersonic Flows Behaviour in a Shock Tube Facility: TVD Schemes and Flux Limiters Application
}

\author{
A. Boulahia, ${ }^{1}$ S. Abboudi ${ }^{2 \dagger}$ and M. Belkhiri ${ }^{3}$ \\ ${ }^{1}$ Mechanical Engineering department, Larbi Ben M'hidi University , Oum El Bouaghi, Algeria \\ ${ }^{2}$ IRTES Institute, M3M Laboratory, site de Sévenans, University of Technology of Belfort-Montbéliard (UTBM), France \\ ${ }^{3}$ Energy Physics Laboratory, University of MENTOURI, Constantine, Algeria \\ †Corresponding Author Email: said.abboudi@utbm.fr
}

(Received January 10, 2013; accepted April 3, 2013)

\begin{abstract}
Work performed in this study concerns mainly the analysis and the wisely use of TVD type schemes (total variation diminishing) for numerical simulation of reactive flows, these schemes are first presented in scalar equation. Their extension to Euler equations for a reactive gas mixture is conducted through the approximate extended solver of Riemann problem. A comparative study of specific variants of TVD schemes has been made in the case of one-dimensional unsteady flow for an inert and reactive gas mixture, which represents the classical instance of a shock tube. The purpose of this investigation is to highlight the general behaviour (order of accuracy) and performance of TVD schemes with various flux limiters for the simulation of reactive flows and in particular, to make possible the capture of the shock wave together with waves expansion for choosing the appropriate scheme to apply eventually in simulation of hypersonic viscous flow in chemical non equilibrium.
\end{abstract}

Keywords: Shock waves, Hypersonic flows, Reactive flows, TVD schemes, Flux limiter, Shock tube.

\section{NOMENCLATURE}

$\begin{array}{ll}F & \text { Numerical flux } \\ u, v & \text { Velocity components } \\ S & \text { Vectors of sources termes } \\ R & \text { Matrix of eigen values } \\ h_{i} & \text { Yee numerical flux } \\ x, y & \text { Directions } \\ Q_{i} & \text { Centred scheme of limiter Function } \\ P & \text { Pressure [Pa] } \\ E & \text { total Energy of gas mixture } \\ e & \text { Internal Energy of gas mixture }\left[\mathrm{m}^{2} \mathrm{~s}^{-2}\right] \\ T & \text { Temperature [K] } \\ U & \text { Vector of conservative variables } \\ q & \text { Variable : Newton }- \text { Raphson } \\ f & \text { Hyperbolic scalar function } \\ a & \text { Eigenvalues of } A\end{array}$

$\begin{array}{ll}i & \text { Mesh point index } \\ z & \text { Space variable } \\ A & \text { Flux Jacobian Matrix } \\ t & \text { Time Variable } \\ \Phi & \text { Vector of heat flux } \\ \alpha_{\mathrm{x}} & \text { Parameter equal to } R_{x_{i+1 / 2 j}^{-1}}\left(U_{i+1, j}+U_{i, j}\right) \\ \sigma & \text { Parameter equal to } 1 / 2 \psi(x) \\ \delta & \text { Correction parameter } \psi(z) \\ \rho & \text { Density [ Kg.m }{ }^{-3} \text { ] } \\ \psi & \text { Entropic correction function } \\ \phi & \text { Dissipation function } \\ \Delta & \text { Difference between two elements } \\ \gamma & \text { Specific heat ratio } \\ \lambda & \text { Time / space ratio } \\ \Delta_{\mathrm{i}} & \text { Parameter equal to }\left(u_{i+1}-u_{i}\right)\end{array}$




\section{INTRODUCTION}

When treating numerically the fluid mechanic equations, the necessity of computing convective fluxes arises. Generally, polynomial functions were often used to approach the variations of variables. But this approach becomes inappropriate if the solution contains discontinuities. In these cases, schemes that use second or higher order approximations present inconveniences during the convergence process and solutions have oscillations next to the discontinuity (scheme highly diffusive). To deal with this problem, flux limiter functions were built as linear combinations of first and second order approximations (Sweeby P 1984) . These schemes are known as TVD although, the TVD property has expressly only been demonstrated for scalar convection equations. If we use the first order approach, the resultant scheme is diffusive; on the other hand, if the second order approach has more credibility the scheme is compressive. When extending these concepts from systems of linear equations to non linear, the certainty that they continue being TVD is lost (Toro E 1999).

In some papers ( Comg Yu 2006 ), (Yee 1987 ), and (Yee and al 1985) and books (Laney 1998) and (Anderson 2004), various schemes were proposed, in one hand, to look for the best way to capture contact discontinuities without the characteristic smearing linked to degenerate linear waves, and on the other hand, which have the capacity to solve satisfactorily discontinuities associated with lineally degenerate wave families and without losing robustness in dealing with gas dynamics shock waves.

One of the most popular scheme was introduced by Harten (Harten 1978) using an Artificial Compression Method (ACM) that renders the local slope of the minmod limiter steeper. This last was implemented in second order central difference schemes for Euler equations (Lie and al 2003) More recent ACM applications are given in Ref. (Lo and al 2007).

The construction of a large number of second order TVD schemes is based essentially on the principle of flux limitation (Hirsch 1992 ), (Queiroz and al 2008). The second order is obtained by adding an anti dissipative flux that should be limited to ensure the TVD property. To simulate of reactive flow we have applied a TVD discretization to no stationary Euler equations for a one-dimensional reactive flow to study the influence of the scheme choice and flux limiter. In this work we present first the numerical process used. We then investigate the case of a shock tube containing two different inert gases before presenting results registered in a shock tube containing a mixture of reactive gases

\section{MODELLING PROCESS}

For the case of a two-dimensional flow with an inert gas mixture, Euler equations can be written in their conservative form as follow:

$$
\frac{\partial U}{\partial t}+\frac{\partial F(U)}{\partial x}+\frac{\partial G(U)}{\partial y}=0 .
$$

where $U, F$ and $G$ are vectors expressed as follow:

$$
U=\left(\begin{array}{c}
\rho_{1} \\
\rho_{2} \\
\cdot \\
\cdot \\
\cdot \\
\rho_{n s} \\
\rho u \\
\rho v \\
E
\end{array}\right), F=\left(\begin{array}{c}
\rho_{1} u \\
\rho_{2} u \\
\cdot \\
\cdot \\
\cdot \\
\rho_{n s} u \\
\rho u^{2}+P \\
\rho u v \\
u(E+P)
\end{array}\right), G=\left(\begin{array}{c}
\rho_{1} v \\
\rho_{2} v \\
\cdot \\
\cdot \\
\cdot \\
\rho_{n s} v \\
\rho u v \\
\rho v^{2}+P \\
v(E+P)
\end{array}\right) .
$$

There are different approaches to treat the problem of an inert or reactive gas mixture. If at first, we ignore the diffusion phenomena, convection pressure phenomena are described by the momentum, total energy and the Euler equations for the density of the gas mixture. The evolution of species is represented by the equations of balance sheet, or for an inert gas mixture by a convection equation for the partial densities, the coupling between the two processes is performed by the equation of mass conservation, but also through the temperature that intervenes in the equation of energy conservation and in the calculation of chemical production rate if the gas mixture is reactive. The conceived algorithms are based on the discretization methods developed in the non-reactive case (without source terms). Whereas the source terms presence is the origin of new phenomena (such a strong coupling between characteristics equations), not taken into account by these methods (Perrel and al 1990 ) The numerical method implemented here deals with the fully coupled set of the problem equations. Thus, the discretization scheme relies on an extension of Riemann solvers obtained in the case of an ideal gas applied to a gas mixture which has been emitted by Roe (Roe 1981) and extended by Montagné and Vinokur (Vinokur and al 1990) to a reactive gas mixture.

\section{NUMERICAL DISCRETIZATION}

The discretization of the two-dimensional system of equations (1) is performed by simply addressing in separate manner each of the two directions of space.A second-order accurate in the TV-L1 norm for general smooth and non monotone solutions of total variation diminishing (TVD) finite-difference scheme is then applied to the discretization of the flux $F$. The numerical procedure implemented here deals specifically with the computation of an unsteady solution for which we have chosen an explicit discretization accurate to the second order in space and time for the convective terms. To overcome stiffness due to source terms, these last are discretized implicitly. Therefore, by introducing $\Delta x$ and $\Delta y$ as space steps such that $x_{i}=(i-1) \Delta x$ and $y_{i}=(i-1) \Delta y$ we obtain an equation of the form: 


$$
U_{i}^{n+1}-U_{i}^{n}+(\Delta t / \Delta x)\left(\tilde{F}_{i+1 / 2}^{n}-\tilde{F}_{i-1 / 2}^{n}\right)=\Delta t S_{i}^{n+1}
$$

Where we take $v=0$ and remove the $(n s+2)^{\text {th }}$ component. The expression of the flux $\tilde{F}_{i+1 / 2}^{n}$ (not taking into account the $\mathrm{j}$ index) is:

$$
\tilde{F}_{i+1 / 2, j}=0.5\left(F_{i, j}+F_{i+1, j}+R_{x_{i+1 / 2, j}} \Phi_{x_{i+1 / 2, j}}\right)
$$

The $\phi_{x}^{l}$ components of $\Phi_{x}$ vectors are given for decentred upwind TVD schemes or symmetrical by the following identities:

$$
\begin{aligned}
& \phi_{x_{i+1 / 2, j}}^{l^{U}}=\sigma\left(a_{x_{i+1 / 2, j}}^{l}\right)\left(g_{x_{i+1 / 2, j}}^{l}+g_{x_{i, j}}^{l}\right) \\
& -\psi\left(a_{x_{i+1 / 2, j}}^{l}+\gamma_{x_{i+1 / 2, j}}^{l}\right) \alpha_{x_{i+1 / 2, j}}^{l} \\
& \phi_{x_{i+1 / 2, j}}^{l^{s}}=-\psi\left(a_{x_{i+1 / 2, j}}^{l}\right)\left(\alpha_{x_{i+1 / 2, j}^{l}}^{l}-\tilde{Q}_{x_{i+1 / 2, j}}^{l}\right) \\
& \text { With } \sigma(z)=0.5\left[\psi(z)-(\Delta t / \Delta x) z^{2}\right] \text { and } \\
& \alpha_{x_{i+1 / 2, j}}=R_{x_{i+1 / 2, j}}^{-1}\left(U_{i+1, j}+U_{i, j}\right) \text {. And for a }
\end{aligned}
$$

symmetrical scheme of the $\Phi_{i+1 / 2}$ components are:

$$
\begin{aligned}
& \phi_{x_{i+1 / 2, j}}^{l^{s}}=-(\Delta t / \Delta x)\left(a_{x_{i+1 / 2, j}}^{l}\right)^{2} \tilde{Q}_{x_{i+1 / 2, j}}^{l}- \\
& \psi\left(a_{x_{i+1 / 2, j}^{l}}^{l}\right)\left(\alpha_{x_{i+1 / 2, j}}^{l}-\widetilde{Q}_{x_{i+1 / 2, j}}^{l}\right)
\end{aligned}
$$

For unsteady applications, the entropic parameter $\delta_{1}$ involved in the function $\psi$ can be set to zero:

$$
\psi(z)=\left\{\begin{array}{cl}
|x| & |x| \geq \delta, \\
\left(z^{2}+\delta^{2}\right) / 2 \delta, & |x|<\delta .
\end{array}\right.
$$

The non-linear system is solved by employing the method of Newton - Raphson: $\lim _{m} q^{m}=U^{n+1}$ and the source term is linearised around $q^{m}$ :

$$
S_{i}^{m+1}=S_{i}^{m}+(\partial S / \partial U)_{i}^{m}\left(q_{i}^{m+1}-q_{i}^{m}\right) .
$$

Thus, we get from (3) the following discretization:

$$
\begin{gathered}
\left(I-\Delta t(\partial S / \partial U)_{i}^{m}\right)\left(q_{i}^{m+1}-q_{i}^{m}\right)=-\left[\left(q_{i}^{m}-U_{i}^{m}\right)\right. \\
\left.+(\Delta t / \Delta x)\left(\tilde{F}_{i+1 / 2}^{m}-\tilde{F}_{i-1 / 2}^{m}\right)\right]+\Delta t S_{i}^{m}
\end{gathered}
$$

Solving (9) involves the inversion of a matrix of (ns $x$ ns) dimension, because the last two rows of the matrix $\partial S / \partial U$ are zero. We can also reduce the size of the system by considering the property of conservation of atomic elements (Wuthrich and al. 1994)

\subsection{Flux limiters and TVD schemes}

Here we present a unified formulation of an accurate class of TVD schemes to second order made by Yee (Yee 1989) and in which the flux $h_{i+1 / 2}$ is in general expressed as the sum of a centred term and a dissipation term:

$$
h_{i+1 / 2}=0.5\left(f_{i+1}+f_{i}+\phi_{i+1 / 2}\right)
$$

Schemes that we have taken into account in this study and which we are going to explain briefly in what follows differ only by the expression of the $\phi$ function

\subsection{Finite difference $2^{\text {nd }}$ order decentred upwind scheme of Harten - Yee}

Yee (Yee and al 1987) has proposed a less dissipative scheme of Harten second order by factoring the term $\sigma\left(a_{i+1 / 2}\right)$. Thus, she gets a scheme that is TVD, only for constant coefficients, with very good results. The $\phi$ function is then written as:

$\phi_{i+1 / 2}=\sigma\left(a_{i+1 / 2}\right)\left(g_{i+1}-g_{i}\right)-\psi\left(a_{i+1 / 2}+\gamma_{i+1 / 2}\right) \Delta_{i+1 / 2}(11)$

The limiter function $\mathrm{g}_{i}$ can be one of the following:

- minmod of Roe ( Roe and al 1991)

$$
\begin{aligned}
& g_{i}=\min \bmod \left(\Delta_{i+1 / 2}, \Delta_{i-1 / 2}\right) \\
& \text { - Van Leer } \quad(\text { Van Leer 1974) } \\
& \left.g_{i}=\left(\Delta_{i+1 / 2} \Delta_{i-1 / 2}+\mid \Delta_{i+1 / 2} \Delta_{i-1 / 2}\right)\right) /\left(\Delta_{i+1 / 2}+\Delta_{i-1 / 2}\right) \\
& \text { - Van Albada } \quad(\text { Van Albada and al 1982) } \\
& g_{i}=\frac{\left.\left(\Delta_{i+1 / 2}\left\lfloor\left(\Delta_{i-1 / 2}\right)^{2}+\delta_{2}\right\rfloor+\Delta_{i-1 / 2} \mid\left(\Delta_{i+1 / 2}\right)^{2}+\delta_{2}\right\rfloor\right)}{\left(\left(\Delta_{i+1 / 2}\right)^{2}+\left(\Delta_{i-1 / 2}\right)^{2}+2 \delta_{2}\right)}
\end{aligned}
$$

- Collela (Woodwars and al 1984 )

$g_{i}=\min \bmod \left(2 \Delta_{i-1 / 2}, 2 \Delta_{i+1 / 2}, 0.5\left(\Delta_{i-1 / 2}+\Delta_{i+1 / 2}\right)\right)$

- Superbee ( Roe 1985)

$$
g_{i}=S \max \left[0, \min \left(2\left|\Delta_{i+1 / 2}\right|, S \Delta_{i-1 / 2}\right), \min \left(\left|\Delta_{i+1 / 2}\right|, 2 S \Delta_{i-1 / 2}\right)\right]
$$

Where $S=\operatorname{sign}\left(\Delta_{i+1 / 2}\right)$. If $\left(\Delta_{i-1 / 2}+\Delta_{i+1 / 2}\right)=0$, we take $g_{i}=0$ in Eq. (13), $\delta_{2}$ [Eq. (14)] is a parameter whose sole purpose is to prevent division by zero.

$\operatorname{minmod}\left(x_{1}, \ldots, x_{k}\right)=\operatorname{sign}\left(x_{1}\right) \max \left(0, \max \left(\left|x_{1}\right|, x_{2} \operatorname{sign}\left(x_{1}\right), \ldots, x_{k} \operatorname{sign}\left(x_{1}\right)\right)\right)$

\section{3. finite difference $2^{\text {nd }}$ order symmetrical scheme of Yee- Roe- Davis}

The previous scheme is a decentred scheme in the sense that the dissipation term $\phi_{i+1 / 2}$ depends on the sign of the characteristic velocity. In the following scheme, the dissipation does not depend on the sign of the characteristic velocity. The resulting scheme in this case is somewhat more diffuse, but its implementation is less expensive. Thus, the $\phi$ can be expressed by (Yee 1984):

$$
\phi_{i+\frac{1}{2}}=-\psi\left(a_{i+\frac{1}{2}}\right)\left(\Delta_{i+\frac{1}{2}}-\tilde{Q}_{i+\frac{1}{2}}\right)
$$

Here Again, several choices are possible for the limiter function $\tilde{Q}_{i+\frac{1}{2}}$, including:

$\tilde{Q}_{i+\frac{1}{2}}=\min \bmod \left(\Delta_{i+\frac{1}{2}}, \Delta_{i-\frac{1}{2}}\right)+\min \bmod \left(\Delta_{i+\frac{1}{2}}, \Delta_{i-\frac{3}{2}}\right)-\Delta_{i+\frac{1}{2}}$

$\tilde{Q}_{i+\frac{1}{2}}=\min \bmod \left(\Delta_{i-\frac{1}{2}}, \Delta_{i+\frac{1}{2}}, \Delta_{i-\frac{3}{2}}\right)$

$\tilde{Q}_{i+\frac{1}{2}}=\min \bmod \left(2 \Delta_{i-\frac{1}{2}}, 2 \Delta_{i+\frac{1}{2}}, 2 \Delta_{i+\frac{3}{2}}, \frac{1}{2}\left(\Delta_{i-\frac{1}{2}}-\Delta_{i+\frac{3}{2}}\right)\right)$

Since the simulations are run in unsteady mode, the residual tracking has not been used as a convergence criterion, but computations have been run for long enough (typically up to 1000 iterations, using a CFL limit number up to 0.5 ) to insure 
stability of explicit schemes and to let the flow fully establish.

\section{RESULTS AND INTERPRETATIONS}

\subsection{Mixture of non - reactive gas}

Consider first the case of a shock tube containing two ideal inert gases which their isentropic coefficients are respectively $\gamma_{1}=1.4$ and $\gamma_{2}=1.2$, the initial conditions are described in Table 1 , where the index $L$ (resp. $R$ ) denotes the state of gas in the driver (resp. driven) shock tube. The length of the tube is set at $1 \mathrm{~m}$; and a spatial discretization with constant step in 120 intervals is used.

Table 1. Computing conditions (inert gas mixture)

\begin{tabular}{|c|c|c|c|c|c|c|c|}
\hline$\rho_{I L}$ & $\rho_{I R}$ & $\rho_{2 L}$ & $\rho_{2 R}$ & $P_{L}$ & $P_{R}$ & $u_{L}$ & $u_{R}$ \\
\hline 1.0 & 0.0 & 0.0 & 0.125 & 1.0 & 0.1 & 0.0 & 0.0 \\
\hline
\end{tabular}

Due to the axial symmetry of the shock tube, the flow domain is modelled with an axi- symmetric structured mesh with grid adaptation to resolve regions with the steepest gradients. The axisymmetric approach is appropriate for the cylindrical geometry of the shock tube and is sufficient to render an accurate description of the real flow configuration.

Only the solid boundary condition is considered in the current work since the flow is confined within the tube. At the wall, no slip boundary condition is imposed for the momentum equations to enforce no mass fluxes can penetrate through the solid boundary. For the energy equation, adiabatic condition is assumed. And for Euler equations only a single physical boundary condition is to be imposed. This condition is expressed by the vanishing normal velocity $\left(\mathrm{V}_{\text {normal }}=0\right)$. As a consequence, all convective flux components through the solid boundary will vanish. Uniform grid spacing is used. The boundary conditions of the problem are held fixed as a short time span of the unsteady flow is considered. A uniform time step prescribed by a $C F L$ condition and written as follow, was used throughout the area:

$$
\Delta \tilde{t}_{i, j}=\min \left(\Delta t_{i, j}, \Delta t_{\text {shock }}\right)
$$

Where $\Delta t_{\text {shock }}$ is the maximal value of the time step near the shock and:

$$
\Delta t_{i, j}=\frac{C F L}{\max \left\langle|\operatorname{gradx}|_{i, j}\left(c_{i, j}+u_{i, j}\right),|\operatorname{grady}|_{i, j}\left(c_{i, j}+v_{i, j}\right)\right\rangle}
$$

Finally, Relaxation method by line on $x$ direction was employed to resolve the linear system.

The results of the numerical simulations have been displayed in contour plots. Thus, from the initial time, the wave pattern of this problem consists on the apparition of three waves in the shock tube (Hubert Chanson 2008), separating zones of uniform flow: a relaxation followed by a contact discontinuity and shock. The density profile highlights these three waves (Fig. 1.). The pressure and velocity remain constant across the contact discontinuity while the gas mixture does not vary through the relaxation and the shock wave (Figs. 2, 3 and 4). The exact solution of the problem has been calculated using the method of characteristics (Hubert Chanson 2008), and it is represented in continuous line on all figures.

The exact solution of the problem has been calculated using the method of characteristics. On all figures, this exact solution is represented in dashed line while the numerical solution proposed in this research is indicated by dots.

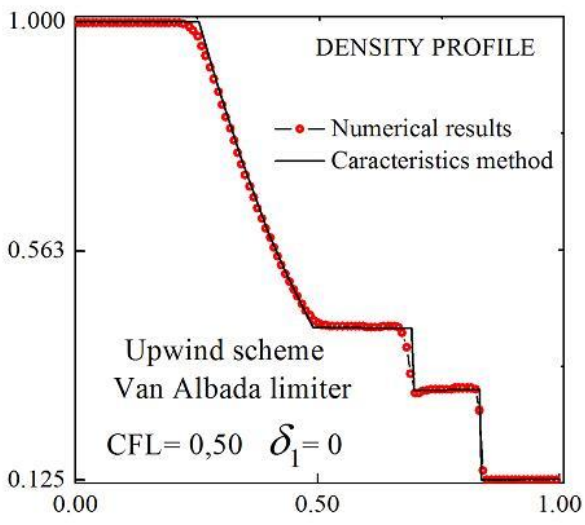

Fig. 1. Shock Tube: an inert gas mixture Density profile, decentred upwind scheme and Van Albada limiter

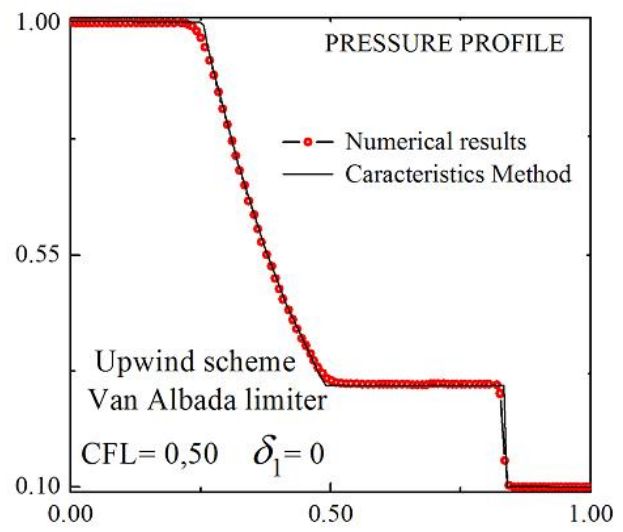

Fig. 2. Shock Tube: an inert gas mixture Pressure profile, decentred upwind scheme and Van Albada limiter

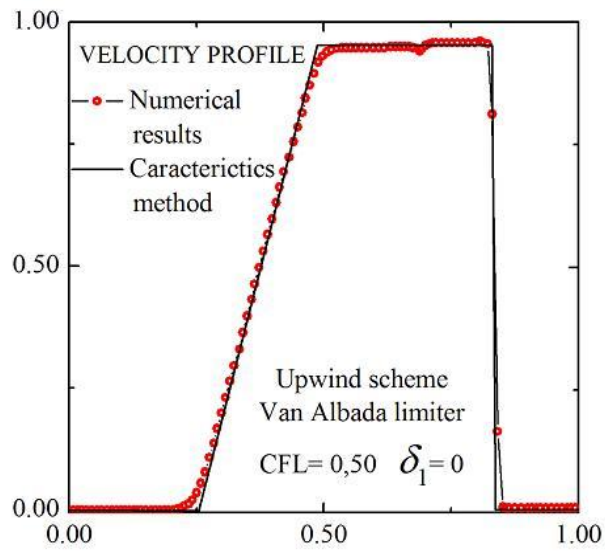

Fig. 3. Shock Tube: an inert gas mixture Temperature profile, decentred upwind scheme and Van Albada limiter 


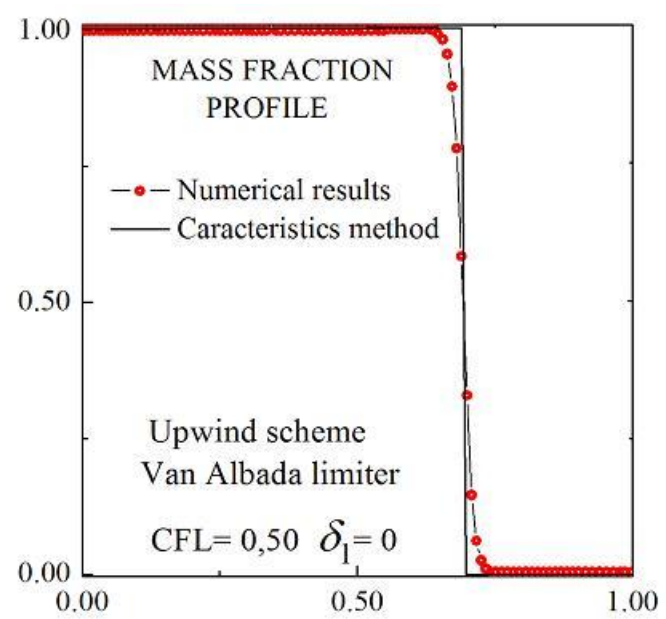

Fig. 4. Shock Tube: an inert gas mixture Mass fraction profile, decentred upwind scheme and Van Albada limiter

\subsection{Study of various schemes}

On Fig. 5 to Fig.7 are represented the density profiles obtained with the symmetrical schemes associated with the three limiters [(18) (19) and (20)]. Likewise, the results obtained with the decentred upwind scheme and five limiters [(12) to (16)] are given in Fig.8 to Fig.11. Limiters here are listed from first to fifth in order of decreasing dissipation.

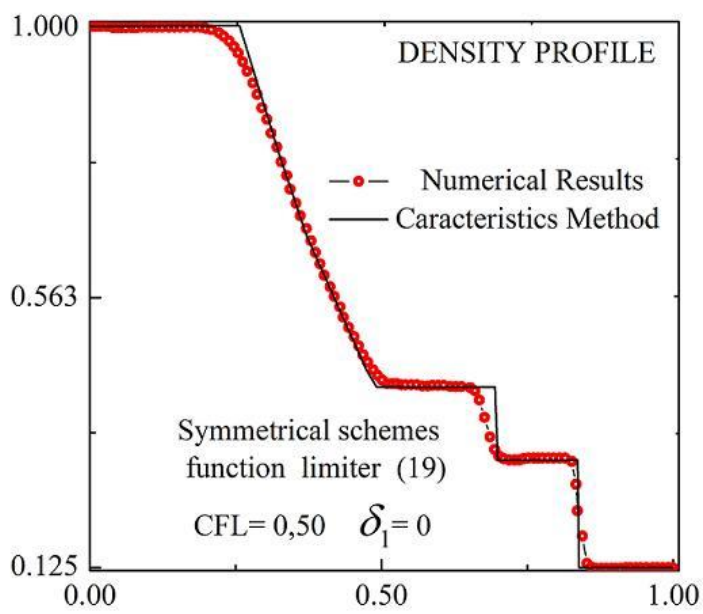

Fig. 5. Shock tube (inert gases mixture):symmetrical scheme and limiters of Roe

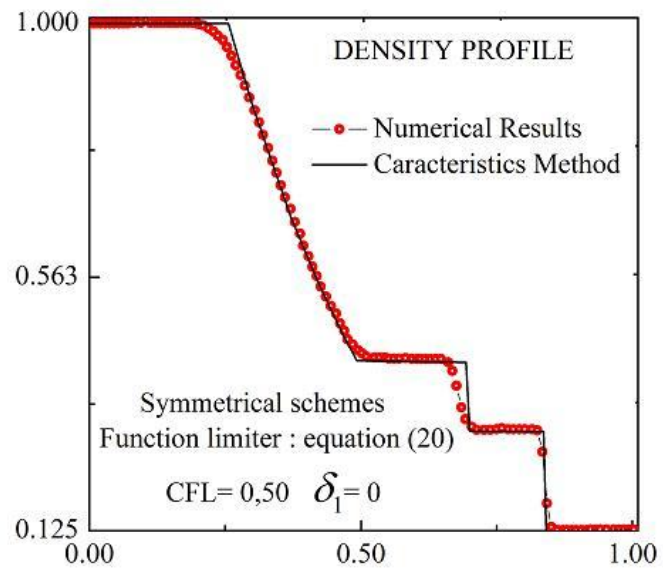

Fig. 6. Shock tube (inert gases mixture):symmetrical scheme and limiters of Van Leer

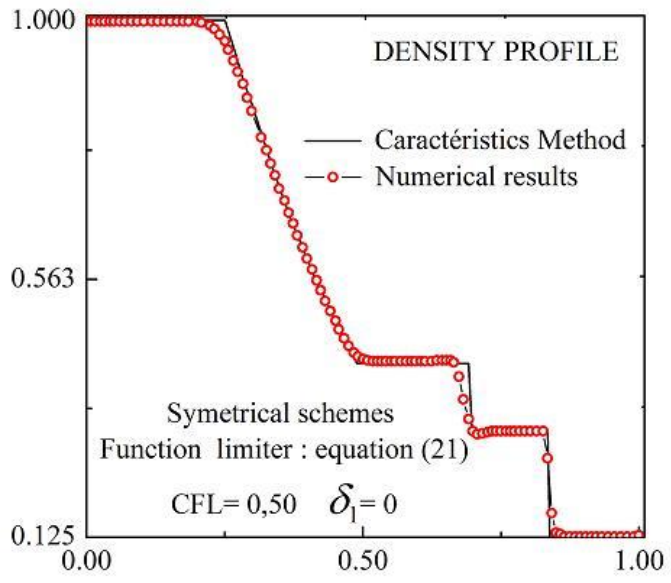

Fig. 7. Shock tube (inert gases mixture): symmetrical scheme and limiter Van Albada

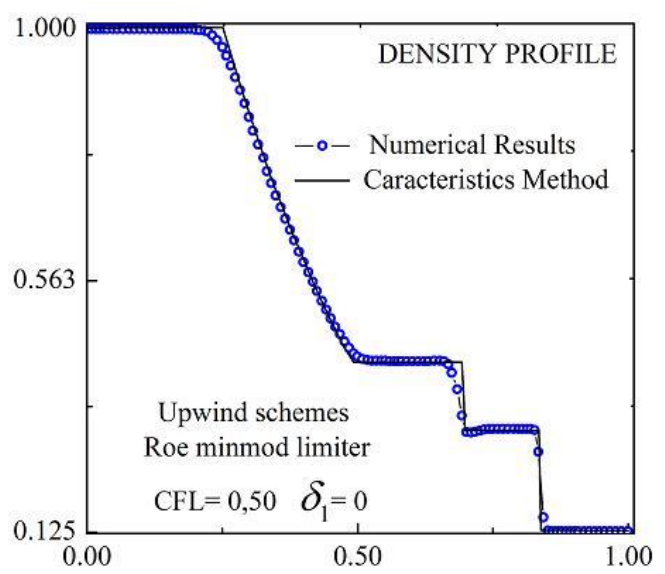

Fig. 8(a). Shock tube (inert gas mixture): decentred upwind scheme and Roe limiter 


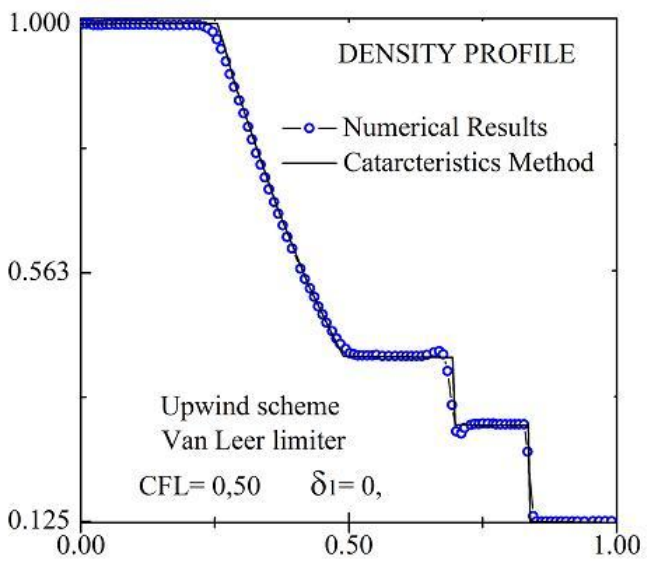

Fig. 8(b). Shock tube (inert gas mixture): decentred upwind scheme and Van Leer limiter

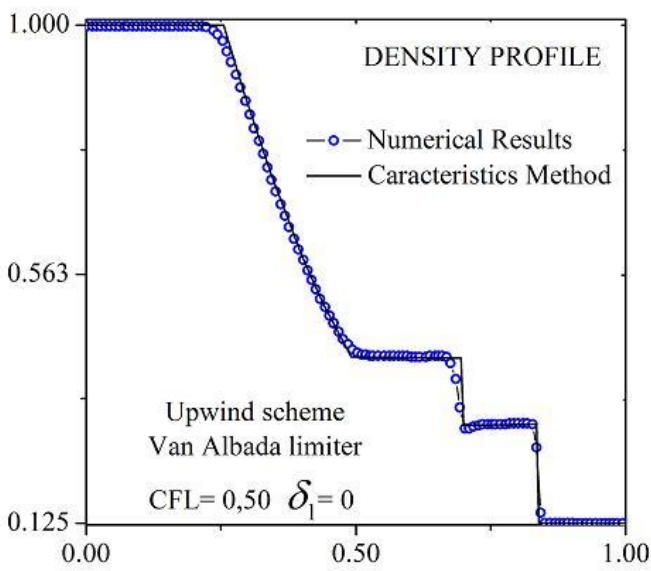

Fig. 9. Shock tube (inert gas mixture): decentred upwind scheme and Van Albada limiter

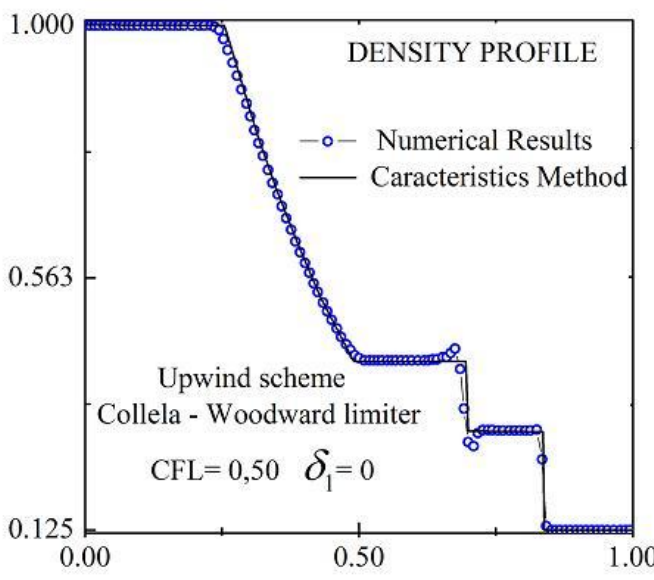

Fig. 10. Shock tube (inert gas mixture): decentred upwind scheme and limiter of Collela - Woodward

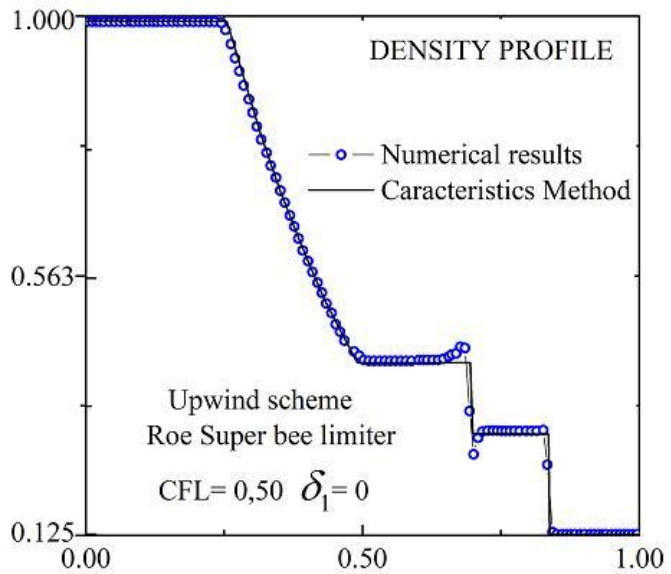

Fig. 11. Shock tube (inert gas mixture): decentred upwind scheme and Super bee limiter

Globally, the symmetrical scheme seems to be more diffusive than the decentred upwind scheme. It enables the shock wave capture at two points, while the capture of the shock wave is slightly more accurate with the decentred upwind scheme. The influence of the limiter choice is most striking about the capture of the shock wave

It appears most clearly in the expansion that the use of the decentred upwind scheme associated with the superbee limiter gives the best results. However, for both types of schemes (symmetrical or decentred upwind) and whatever the chosen limiter, oscillations appear in the vicinity of the contact discontinuity (Laney 1998 ), (Sjögreen. and al 2004) and (Comg Yu 2006)

\subsection{Study of a contact discontinuity}

For the study of the contact discontinuity we have used the numerical flux expression for a scheme of first order in which the entropic correction parameter $\delta$ is equal to zero (Toro 1997):

$$
\tilde{F}_{i+1 / 2}=0.5\left(F_{i}+F_{i+1}-\left|A_{i+1 / 2}\right|\left(U_{i+1 / 2}-U_{i}\right)\right)
$$

Suppose now that at the initial time $t_{0}$, the $i$ and $i$ +1 states are separated by a discontinuity of contact with $u_{L}=u_{R}=u$ and $P_{L}=P_{R}=P$ and through the contact discontinuity we have the following expression data:

$$
U_{i}^{t_{0}}=\left(\begin{array}{c}
0 \\
\rho_{L} \\
\rho_{L} u \\
E_{L}
\end{array}\right) \quad U_{i+1}^{t_{0}}=\left(\begin{array}{c}
\rho_{R} \\
0 \\
\rho_{R} v \\
E_{R}
\end{array}\right)
$$

Suppose further that the speed of propagation of the discontinuity is supersonic, which implies that the scheme is decentred upstream. We then get for the numerical flux $\tilde{F}_{i+1 / 2}=F_{i}$. At the time $t_{0}$, the variables at points $i$ and $i+1$ can be written as follow:

$$
\begin{aligned}
& U_{i}^{t_{1}}=U_{i}^{t_{0}}-\lambda\left(F_{i}-F_{i-1}\right)=U_{i}^{t_{0}} \\
& U_{i+1}^{t_{1}}=U_{i+1}^{t_{0}}-\lambda\left(F_{i+1}-F_{i}\right) .
\end{aligned}
$$

That to say: 


$$
U_{i+1}^{t_{1}}=\left(\begin{array}{c}
(1-\lambda u) \rho_{R} \\
\lambda u \rho_{L} \\
\left(\rho_{R}-\lambda u\left(\rho_{R}-\rho_{L}\right)\right) u \\
(1-\lambda u) E_{R}+\lambda u E_{L}
\end{array}\right)
$$

The density and the velocity at the time $t_{1}$ and at the point of $i+1$ index are given by:

$$
\left\{\begin{array}{l}
\rho_{i+1}^{t_{1}}=\rho_{R}-\lambda u\left(\rho_{R}-\rho_{L}\right) \\
u_{i+1}^{t_{1}}=u
\end{array}\right.
$$

And effectively, we find again the inequality of speeds on both sides of the contact discontinuity. The pressure can be deduced from the expression of total energy: for a mixture of two inert gases whose coefficients $\gamma_{1}$ and $\gamma_{2}$ are constant. We can write:

$$
P=(\gamma-1) \rho e \text { with } \gamma=\frac{\rho_{1} C_{P 1}+\rho_{2} C_{P 2}}{\rho_{1} C_{V 1}+\rho_{2} C_{V 2}}
$$

Thus we can obtain:

$$
\left\{\begin{array}{l}
(\rho e)_{i+1}^{t_{1}}=P\left(\frac{1-\lambda u}{\gamma_{1}-1}+\frac{\lambda u}{\gamma_{2}-1}\right) \\
P_{i+1}^{t_{1}}=P\left(\gamma_{i+1}^{t_{1}}-1\right)\left(\frac{1-\lambda u}{\gamma_{1}-1}+\frac{\lambda u}{\gamma_{2}-1}\right)
\end{array}\right.
$$

Thus we see that for a mixture of gases for which $\gamma_{1} \neq \gamma_{2}$, the pressure at the time $t_{1}$ will be different from the pressure $P$ prevailing on both sides of the discontinuity.

\subsection{Mixture of reactive gas}

We now consider the case where the shock tube (Fig 12) is filled with air, made up of five species $\left(N_{2}, O_{2}, N, O, N O\right)$ that may react chemically. The model of Park (Park 1990) (seventeen reactions) was restrained to calculate chemical reaction velocities. The initial conditions were taken from (Woodward a 1984),(Yee and al 2000) and (Shyen and al 1990). Initially the air is in chemical equilibrium in the two shock tube compartments (driver and driven) and its composition is calculated using the model of Park.

\begin{tabular}{|c|c|c|}
\hline & $R$ & $L$ \\
\hline $\mathrm{P} \quad\left(\mathrm{N} \cdot \mathrm{m}^{-2}\right)$ & $1.1910^{4}$ & $9.9210^{4}$ \\
\hline$(\mathrm{K})$ & 1378 & 4390 \\
\hline e $\quad\left(\mathrm{m}^{2} \cdot \mathrm{s}^{-2}\right)$ & $1.1010^{6}$ & $7.34 \quad 10^{6}$ \\
\hline$\rho_{N 2}\left(\mathrm{Kg} \cdot \mathrm{m}^{3}\right)$ & $2.3010^{-2}$ & $4.9410^{-2}$ \\
\hline$\rho_{O 2}\left(\mathrm{Kg} \cdot \mathrm{m}^{3}\right)$ & $6.9410^{-3}$ & $6.7110^{-4}$ \\
\hline$\rho_{N}\left(\mathrm{Kg} \cdot \mathrm{m}^{3}\right)$ & 0.0 & $2.3410^{-4}$ \\
\hline$\rho_{O}\left(\mathrm{Kg} \cdot \mathrm{m}^{3}\right)$ & $2.0910^{-8}$ & $1.3410^{-2}$ \\
\hline$\rho_{N O}\left(\mathrm{Kg} \cdot \mathrm{m}^{3}\right)$ & $3.3810^{-5}$ & $2.2210^{-3}$ \\
\hline
\end{tabular}
The initial conditions are detailed in Table 2.

Table 2: Calculation Conditions for the shock tube: a mixture of reactive gases
The length of the tube is $1 \mathrm{~m}$ and the mesh is made up of 101 points. All calculations presented in this section were obtained with a $C F L$ equal to 0.21 .

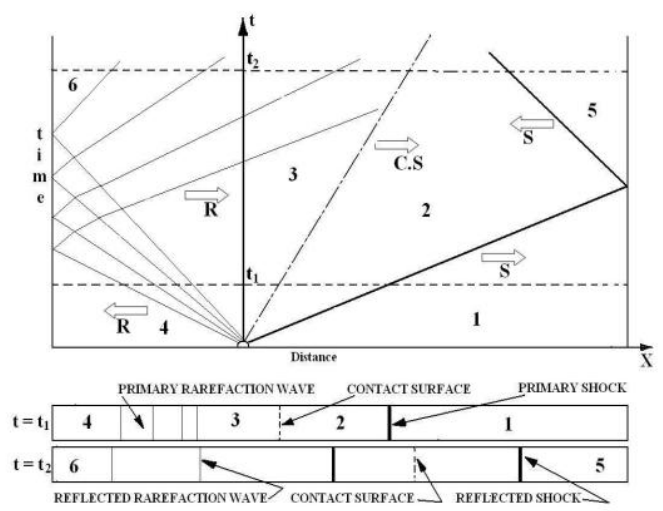

Fig. 12. Shock tube: flow zones and disturbance locations. S: shock wave, CS: contact surface, R: Riemann waves

Fig. 13 to fig. 27. Represent respectively the profiles of density, velocity, temperature, and mass fraction of air species $\left(\mathrm{N}_{2}, \mathrm{O}_{2}, \mathrm{~N}, \mathrm{O}\right.$ and $\left.\mathrm{NO}\right)$ obtained with the symmetrical scheme and the limiters (13) and (14). The same profiles obtained this time with the decentred upwind scheme and limiters (14) and (16) are plotted in fig. 28 to fig. 43

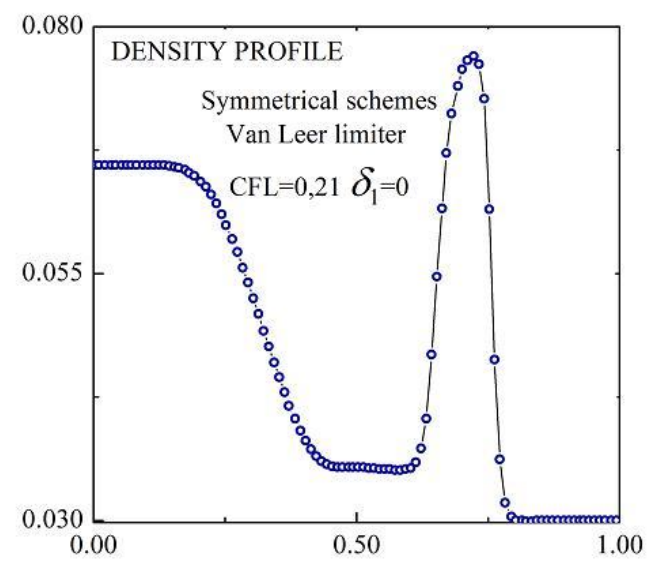

Fig. 13. Shock tube (Reactive gases mixture): Density profiles: (Symmetrical scheme and Van Leer limiter)

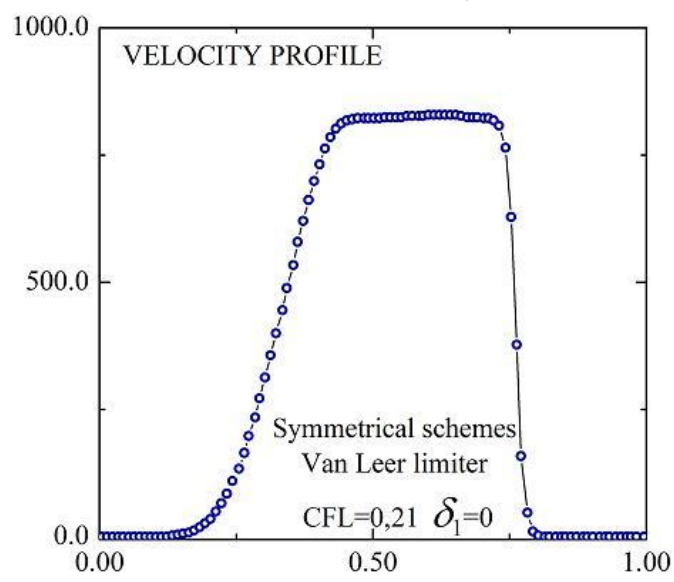

Fig. 14. Shock tube (Reactive gases mixture): velocity profile: (Symmetrical scheme and Van Leer limiter) 


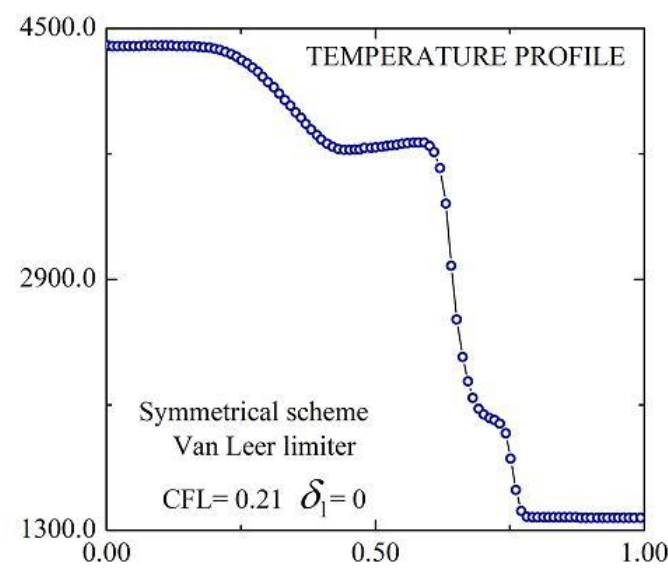

Fig. 15. Shock tube (Reactive gases mixture): temperature profile: (Symmetrical scheme and Van Leer limiter)

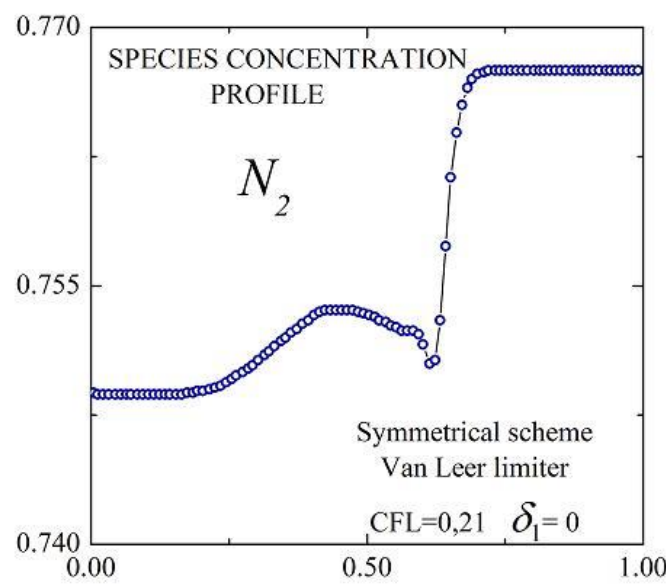

Fig. 16. Shock tube (Reactive gases mixture): mass fraction profile of $\mathrm{N}_{2}$ : (Symmetrical scheme and Van Leer limiter)

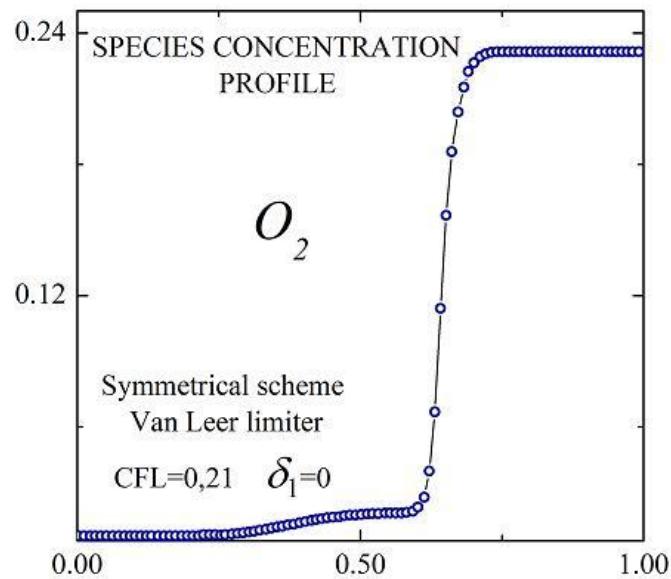

Fig. 17. Shock tube (Reactive gases mixture): mass fraction profile of $\mathrm{O}_{2}$ : (Symmetrical scheme and Van Leer limiter)

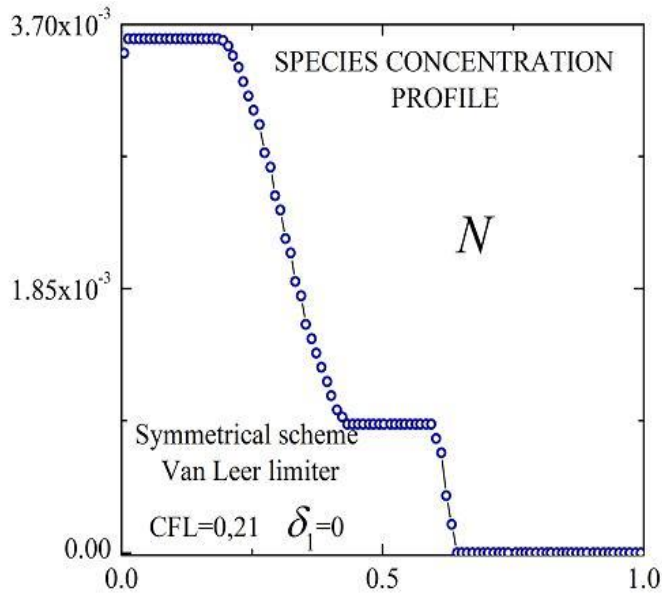

Fig. 18. Shock tube (Reactive gases mixture): mass fraction profile of N: (Symmetrical scheme and Van Leer limiter)

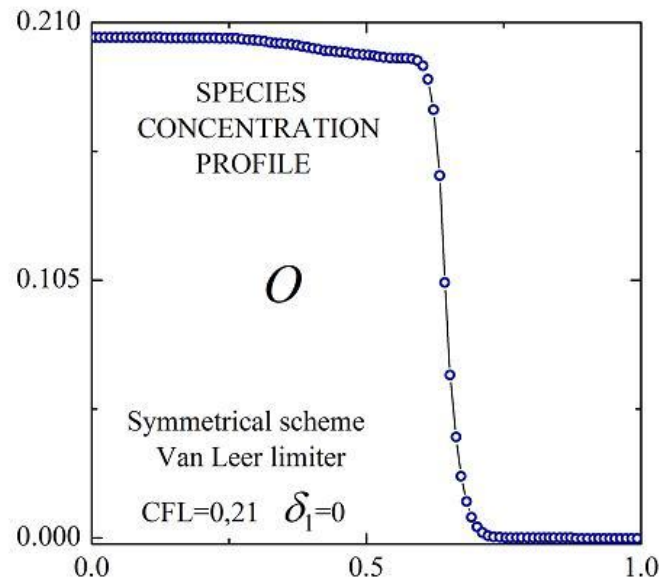

Fig. 19. Shock tube (Reactive gases mixture): mass fraction profile of O: (Symmetrical scheme and Van Leer limiter)

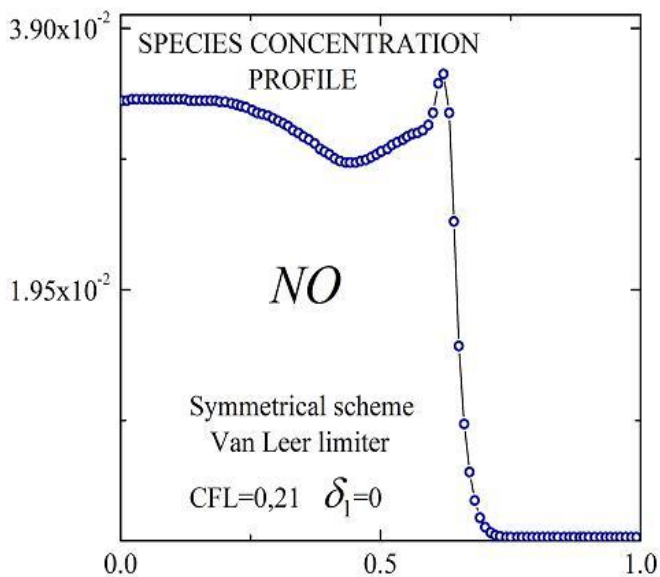

Fig. 20. Shock tube (Reactive gases mixture): mass fraction profiles of NO: (Symmetrical scheme and Van Leer limiter) 


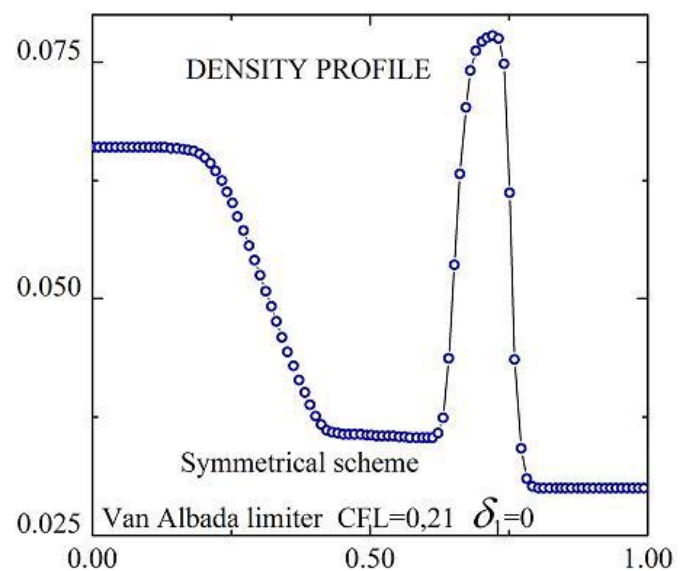

Fig 21. Shock tube (Reactive gases mixture): Density profile: (Symmetrical scheme and Van Albada limiter)

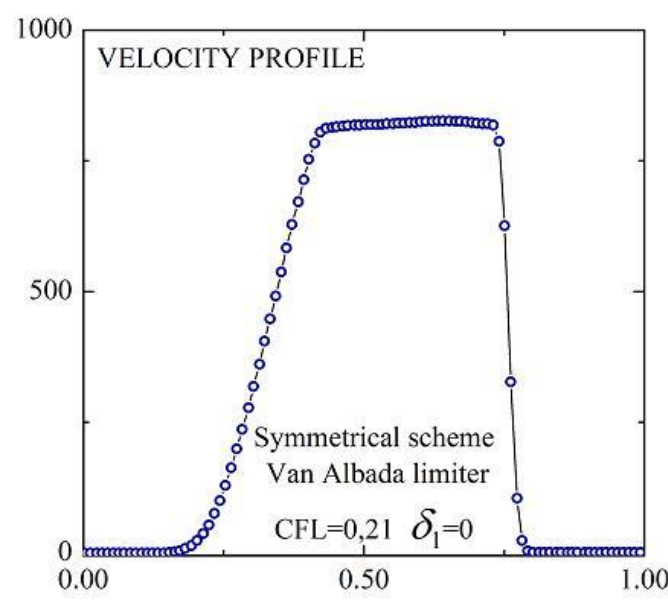

Fig 22. Shock tube (Reactive gases mixture): Velocity profile: (Symmetrical scheme and Van Albada limiter)

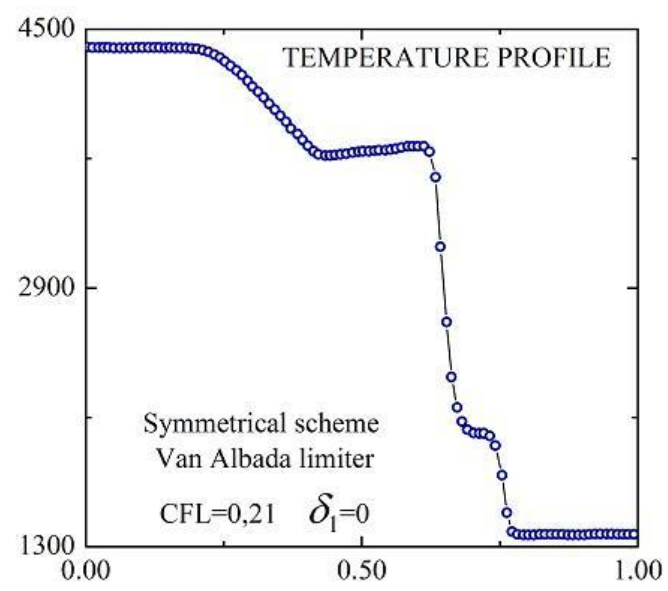

Fig 23. Shock tube (Reactive gases mixture): tempeature profile: (Symmetrical scheme and Van Albada limiter)

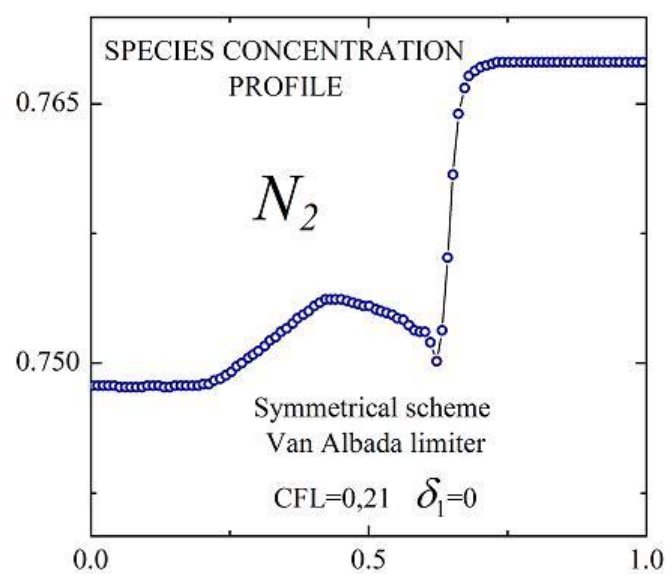

Fig. 24. Shock tube (Reactive gases mixture): mass fraction profile of $\mathrm{N}_{2}$ : (Symmetrical scheme and Van Albada limiter)

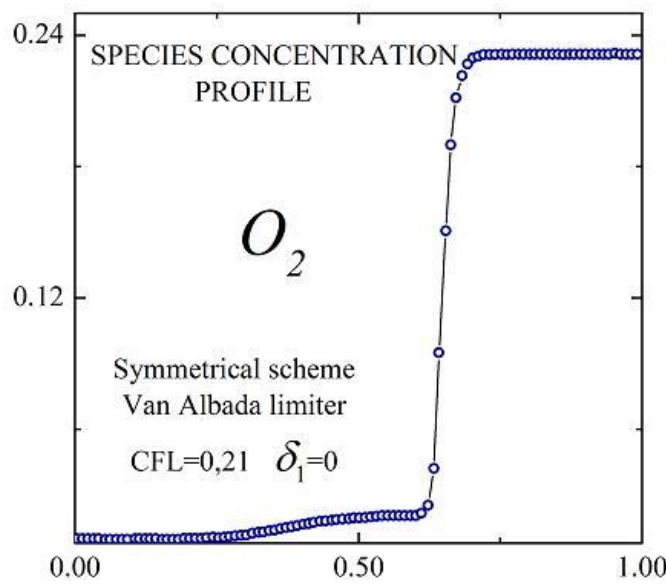

Fig. 25. Shock tube (Reactive gases mixture): mass fraction profile of $\mathrm{O}_{2}$ : (Symmetrical scheme and Van Albada limiter)

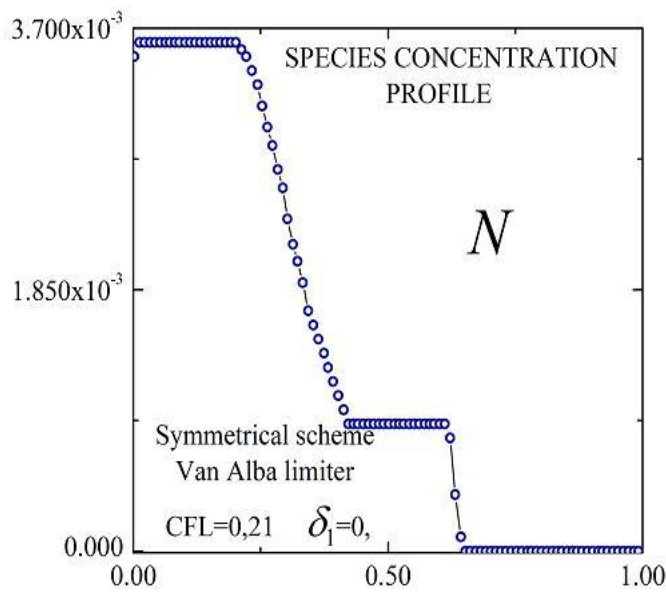

Fig 26. Shock tube (Reactive gases mixture): Mass fraction profile of N: (Symmetrical scheme and Van Albada limiter) 


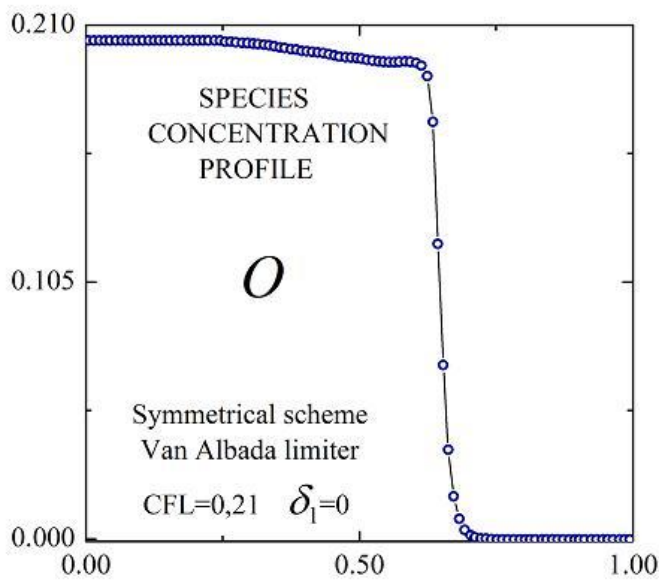

Fig 27. Shock tube (Reactive gases mixture): Mass fraction profile of O: (Symmetrical scheme and Van Albada limiter)

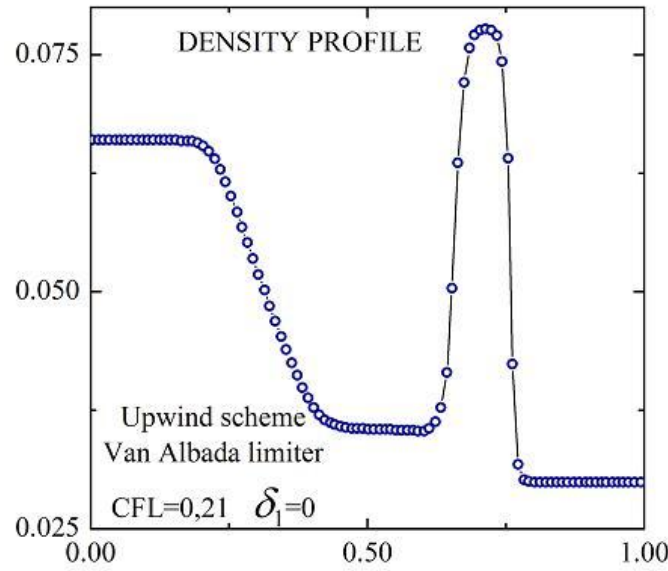

Fig. 28. Shock tube (Reactive gases mixture): Density profile: (decentred upwind scheme and Van Albada limiter)

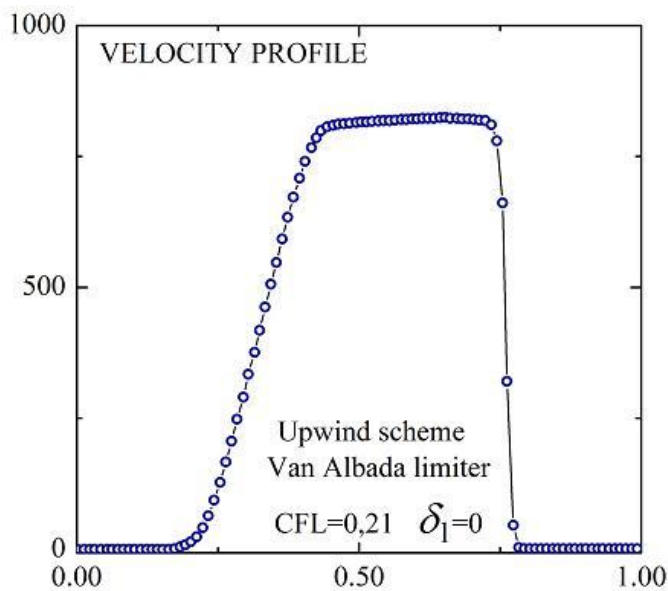

Fig. 29. Shock tube (Reactive gases mixture): Velocity profile: (decentred upwind scheme and Van Albada limiter)

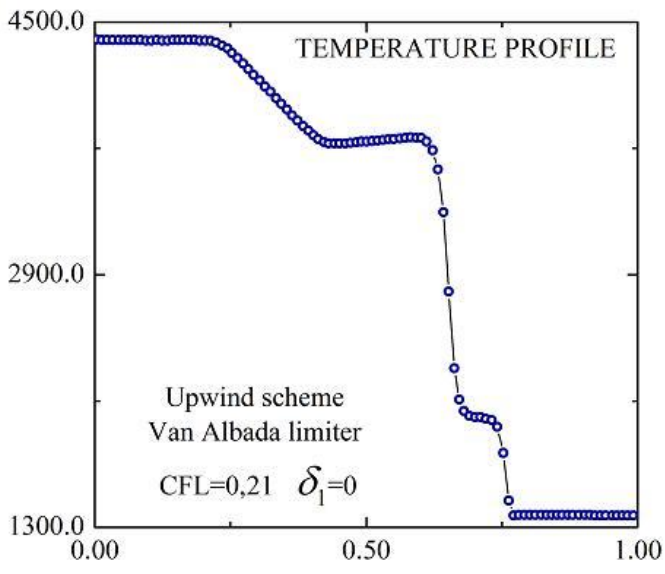

Fig. 30. Shock tube (Reactive gases mixture): temperature profile: (Decentred upwind scheme and Van Albada limiter)

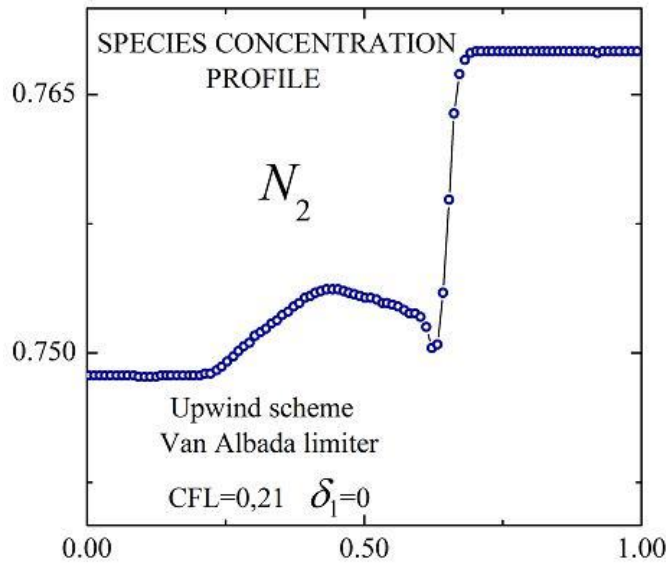

Fig. 31. Shock tube (Reactive gases mixture): mass fraction profile of $\mathrm{N}_{2}$ : (Decentred upwind scheme and Van Albada limiter)

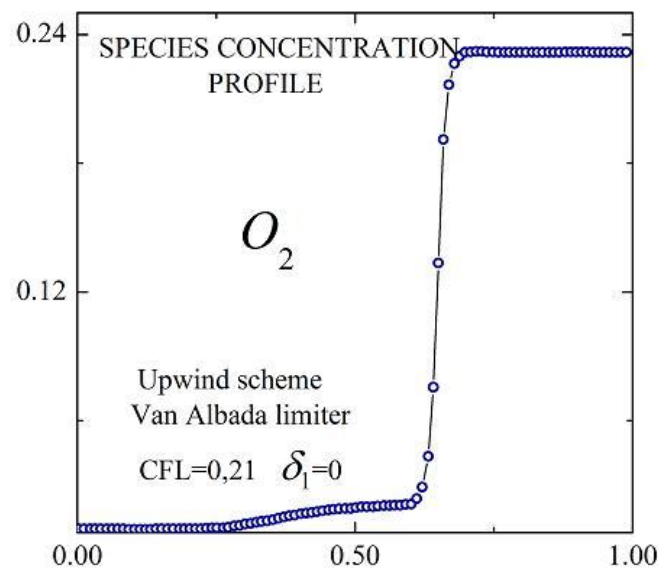

Fig. 32. Shock tube (Reactive gases mixture): mass fraction profile of $\mathrm{O}_{2}$ : (Decentred upwind scheme and Van Albada limiter) 


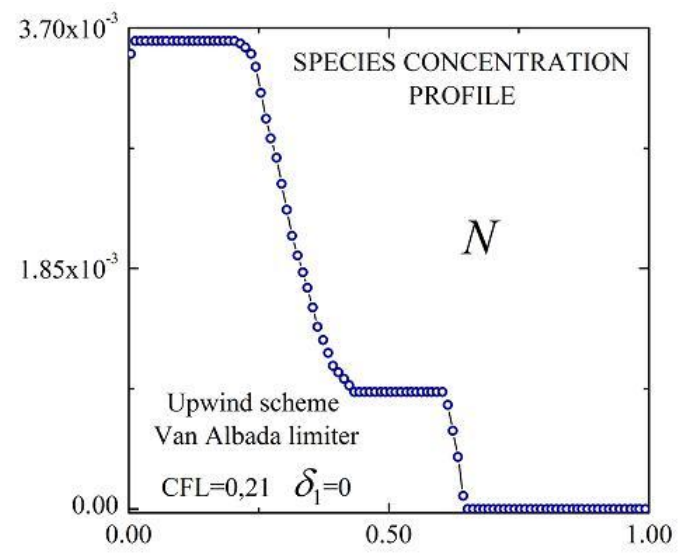

Fig. 33. Shock tube (Reactive gases mixture): mass fraction profile of $\mathrm{N}$ : (Decentred upwind scheme and Van Albada limiter)

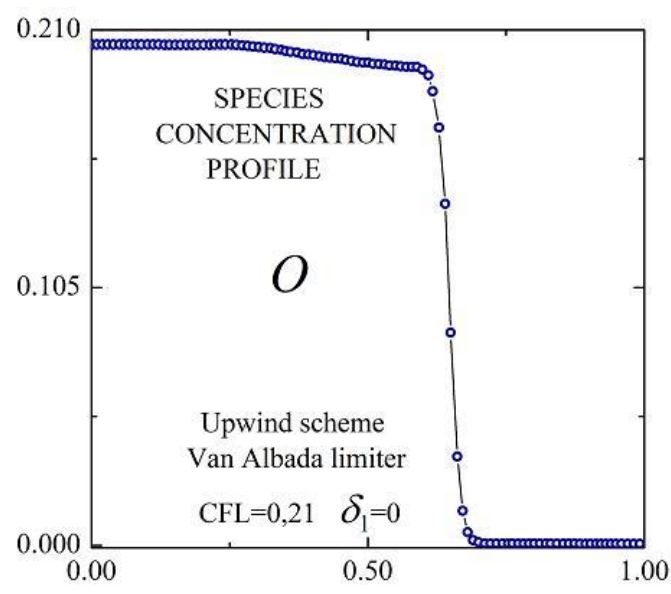

Fig. 34. Shock tube (Reactive gases mixture): mass fraction profile of O: (Decentred upwind scheme and Van Albada limiter)

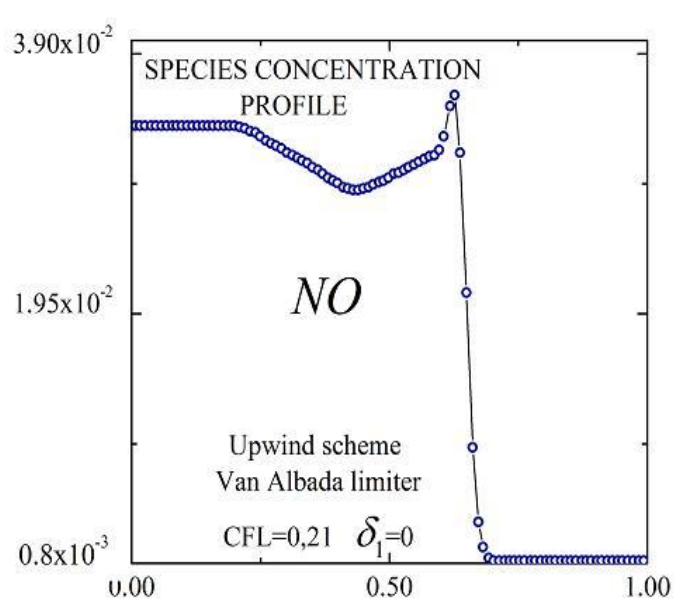

Fig. 35. Shock tube (Reactive gases mixture): mass fraction profile of NO: (Decentred Upwind scheme and Van Albada limiter)

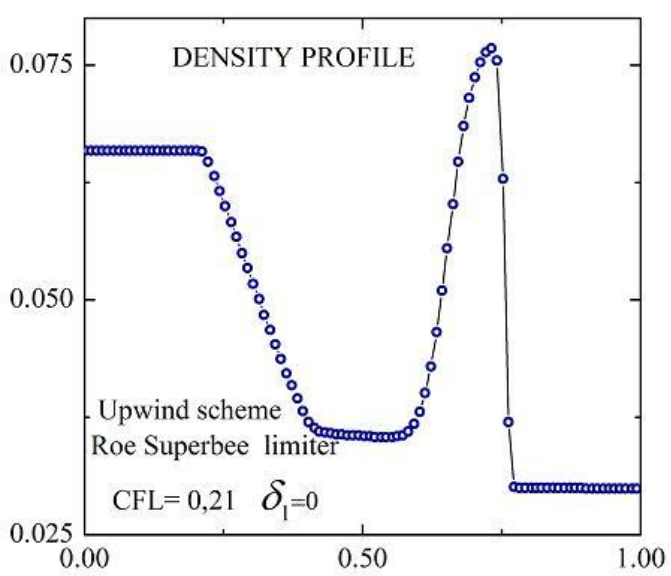

Fig 36. Shock tube (Reactive gases mixture): Density profile: (decentred upwind scheme and Roe-Superbee limiter)

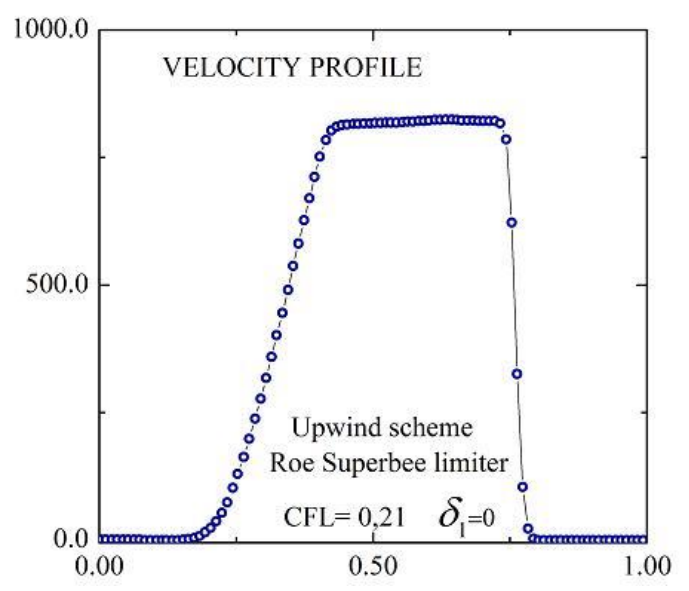

Fig. 37. Shock tube (Reactive gases mixture): Velocity profile: (decentred upwind scheme and Roe-Superbee limiter)

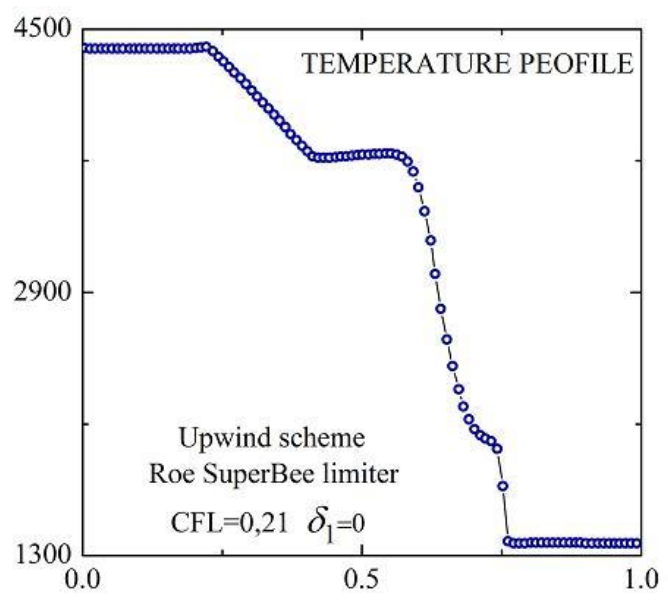

Fig. 38 . Shock tube (Reactive gases mixture): Temperature profile: (Decentred upwind scheme and Roe SuperBee limiter) 


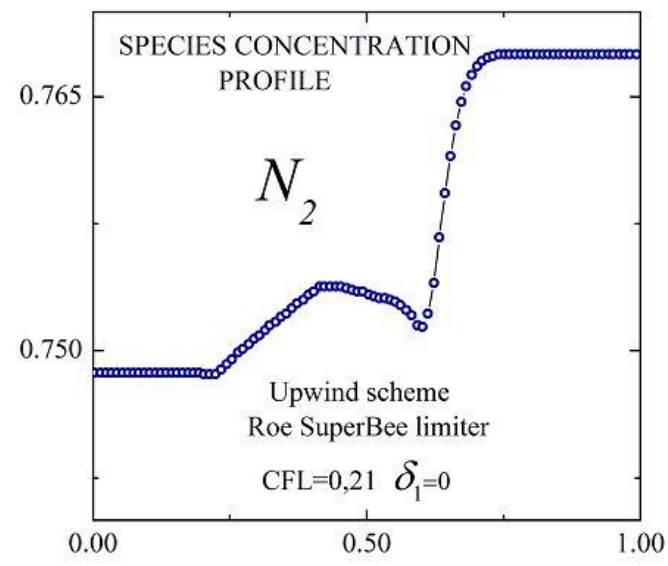

Fig. 39. Shock tube (Reactive gases mixture): Mass fraction of $\mathrm{N}_{2}$ profile: (Decentred upwind scheme and Roe SuperBee limiter)

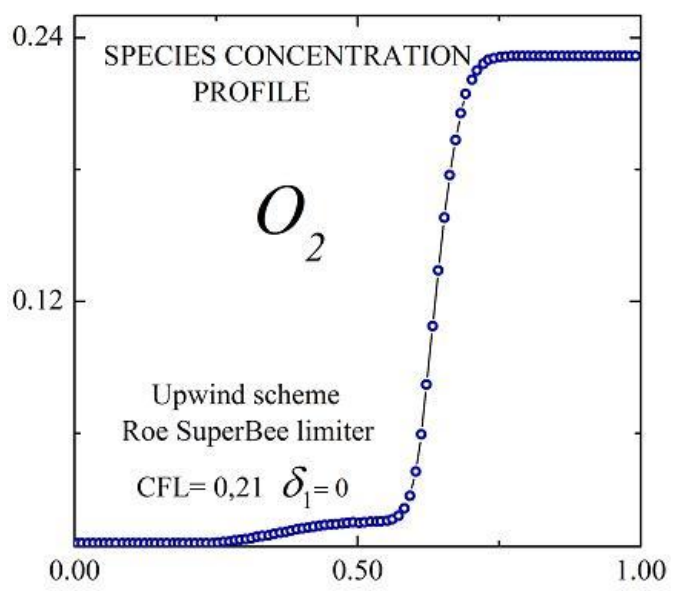

Fig. 40. Shock tube (Reactive gases mixture): Mass fraction of $\mathrm{O}_{2}$ profile: (Decentred upwind scheme and Roe SuperBee limiter)

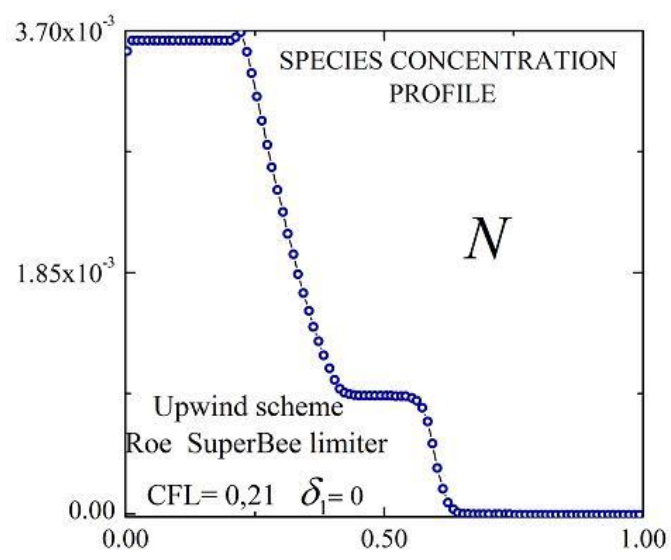

Fig. 41. Shock tube (Reactive gases mixture): Mass fraction of $\mathrm{O}_{2}$ profile: (Decentred upwind scheme and Roe SuperBee limiter)

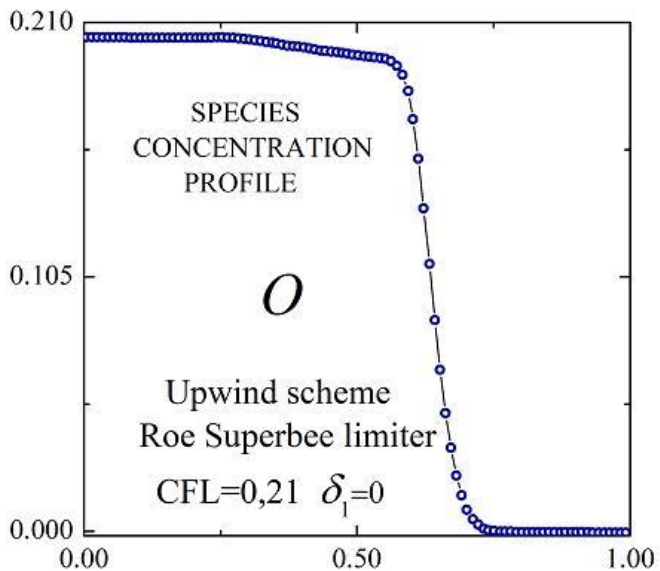

Fig. 42. Shock tube (Reactive gases mixture): Mass fraction of O profile: (Decentred upwind scheme and Roe SuperBee limiter)

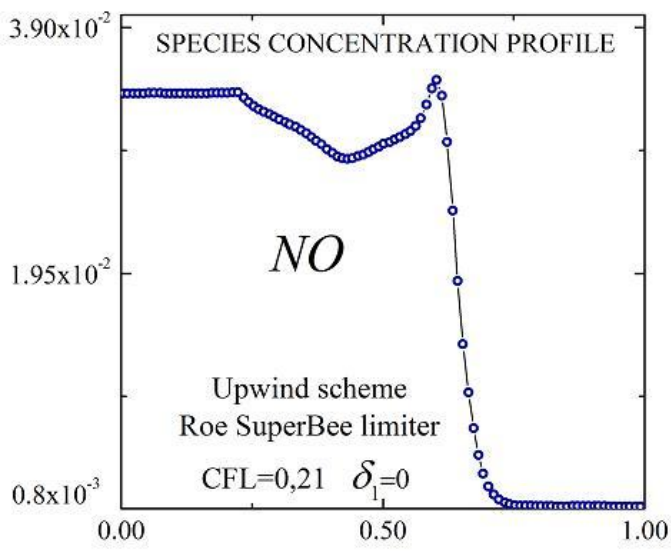

Fig. 43. Shock tube (Reactive gases mixture): Mass fraction of NO profile: (Decentred upwind scheme and Roe SuperBee limiter)

In the case of a reactive gas, the scheme has always the possibility of capturing correctly the three waves. The concentrations remain effectively constant while crossing the shock wave but now they vary during the relaxation due to the presence of source terms. The oscillations at the contact discontinuity registered in this inert gas mixture study still present, and results in a peak on the mass fraction profile of nitrogen monoxide. Indeed, the mass fraction of nitrogen monoxide is a highly nonmonotonous function of temperature and is more stable at intermediate temperatures that appear because of the numerical spreading of the contact discontinuity (Shyen and al.. 1990)

The differences among profiles, which are linked to the choice of the scheme or the limiter, appear mainly at the discontinuity of contact. The amplitude of the oscillation depends on the chosen mass fractions limiter. For example, Superbee flux limiter leads to less marked oscillation but the discontinuity is wider and more spread out.

By keeping the same number of $C F L$, a mesh almost doubled (201 points) was also tested fig. 44 . 


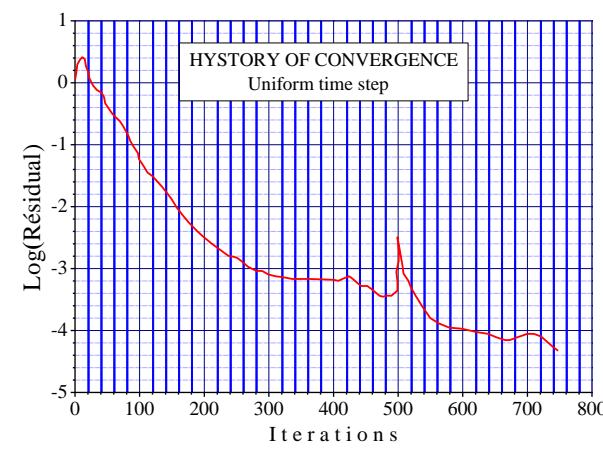

Fig. 44. Convergence History, uniform time step

We verified that the location of the waves is identical to that given by a mesh of 101 points. The resolution is better during the relaxation process and the shock wave includes the same number of interior points. Peaks observed on the mass fractions (concentrations) of nitrogen and nitrogen monoxide are always present. The profiles are plotted in Fig 45 to Fig 47.

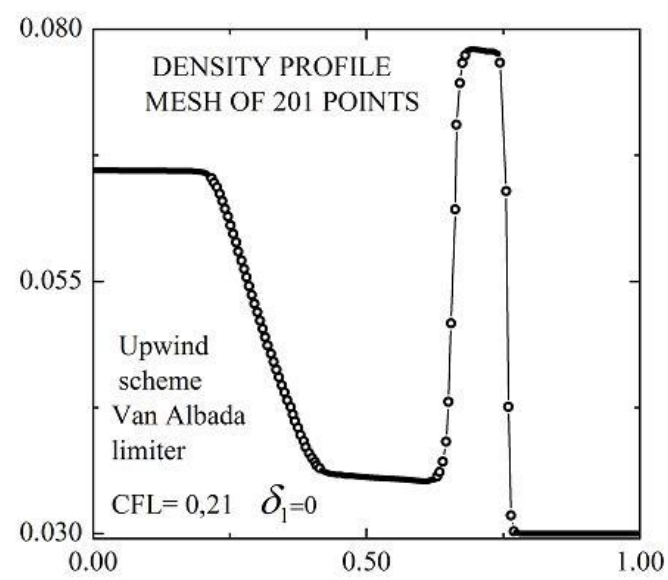

Fig. 45. Reactive gas mixture, Mesh: 201 points Density profile

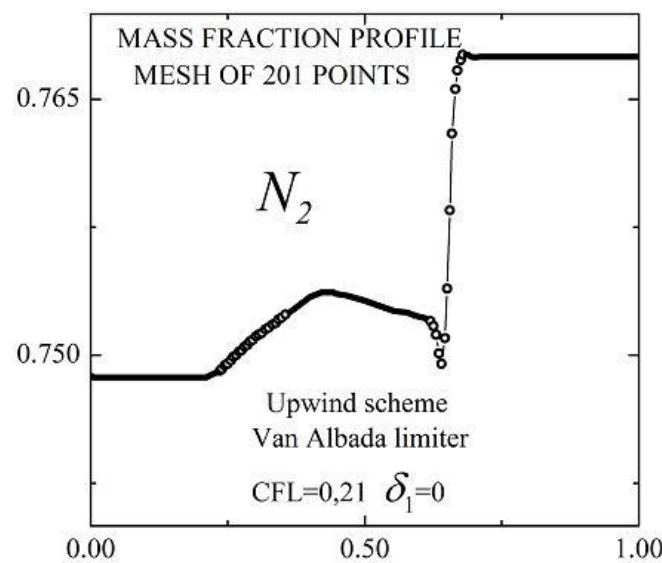

Fig. 46. Reactive gas mixture, Mesh: 201 points Nitrogen molecular profile

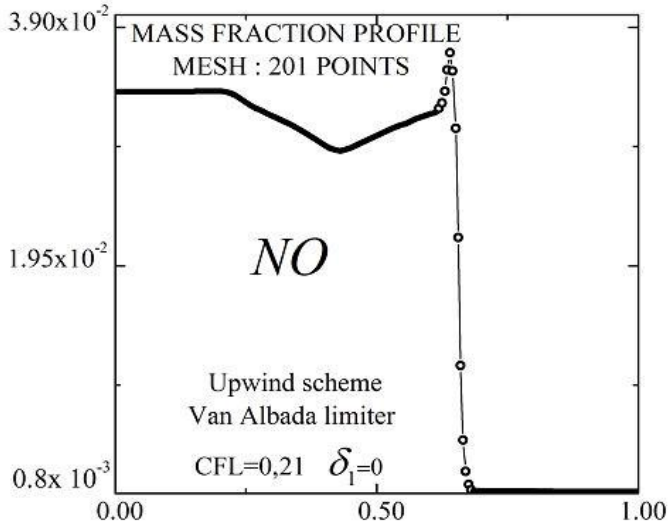

Fig. 47. Reactive gas mixture, Mesh: 201 points Nitrogen molecular profile

\section{CONCLUSION}

This study concerns the application of TVD type schemes with various flux limiters to simulate numerically reactive flows on the basis of the resolution through the Riemann problem solver of Euler equations proposed by Roe and extended by Montagné and Vinokur to this type of flows.

It gave us the opportunity to highlight the correct general behaviour of such schemes and in particular, to evaluate there performances in capturing shock waves. The determination of the beam expansion was performed very satisfactorily. However two difficulties have appeared:

- The first one concern the apparition of just one oscillation of pressure or velocity profiles at the contact discontinuity instead of those issued from other standard schemes: this oscillation, present in both cases of a mixture of inert and reactive gases, is due to the computation of the pressure value in the Roe mean state.

- A second difficulty, own to reactive flows calculations, is linked to the presence of a peak on the mass fractions of nitrogen monoxide and molecular nitrogen near the contact discontinuity: the existence of this peak can be explained by the numerical spreading of the contact discontinuity which causes the appearance of non physical intermediate temperatures for which nitric oxide is stable

On the other hand, for two-dimensional flows applications, a symmetrical scheme (with the Roe min mod flux limiter) was the more accurate among the tested schemes, because its implementation is simpler than that of the decentred upwind scheme and it requires fewer operations. Further, being more dissipative, the symmetrical scheme has proved that it is more stable than the decentred upwind scheme (the value of 0.21 of $C F L$ number was imposed for the stability of the decentred upwind scheme).

\section{ACKNOWLEDGEMENTS}

I would like to thank Professor Said ABBOUDI for his Contribution in writing this paper and for welcoming me in his team. I also thank Professor F.PEYRAUT for welcoming me in his M3M (IRTES) 
Laboratory of University of Technology of BelfortMonbeliard ( UTBM) ., France.

\section{REFERENCES}

Anderson J.D (2004) . Modern Compressible flow with historical perspective .Mc-Graw Hill.

Chanson H (2008). A Simple Solution of the Laminar Dam Break Wave. Journal of Applied Fluid Mechanics JAFM, Vol. 1, No. 1, 1, pp 1-10.

Comg Yu .( 2006). An efficient high resolution shock capturing scheme for multi-dimennsional flows. Chin.J. Astron.Astrophy. vol 6.

Harten A. (1978). The artificial compression method for computation of shocks and contact discontinuities: III self adjusting hybrid schemes Mathematics of Computation 32 363-99.

Hirsch C. (1992) Numerical Computation of Internal and External Flows, Vol.2 Computational Methods for Inviscid and Viscous Flows (London: John Wiley \& Sons Ltd.).

Laney CB (1998). Computational gas dynamics. Cambridge University Press.

Lie K and Noelle S (2003) On the artificial compression method for second-order nonoscillatory central difference schemes for systems of conservation laws SIAM Journal of Scientific Computation 24 1157-74.

Lo S, Blaisdell G and Lyrintzis A (2007) High-order shock capturing schemes for turbulence calculations AIAA Paper $N^{\circ} 2007-827$.

Perrel F, Lafon A. ( 1990) TVD Schemes for a Mixture of Reacting Gases. Technical Report $T R / R F / 14$, CERFACS.

Queiroz R.A.B, Ferreira V.G and Cuenza C.G (2008) A New high resolution TVD scheme for unsteady flows with shock waves . TEMA Tend , Mat, Apl Comput 9, №2-311-320.

Roe P (1981) Approximate Riemann Solvers, parameters vectors and differences schemes. Journal of Comp. Physics, 43: 357-372.

Roe P. (1985) Some contributions to the modelling of discontinuous flows. In Lecture notes in applied Mathematics, Vol 22, Part II, pages 163-193.

Roe P and L. Arora M. Design of Algorithms for a dispersive hyperbolic problem. AIAA $10^{\text {th }}$ CFD conf. Honolulu, Hawai, June 24-27, 1991; AIAA 91-1535.

Shyen SW, Liou MS and Van Leer B. ( 1990) Inviscid flux splitting algorithms for Real gases with non equilibrium chemistry. Jour. of Comp. Physics, 90:371-395.
Sjögreen. B and Yee H. C. (2004) Multiresolution wavelet based adaptive numerical dissipation control for shock-turbulence computation , $J$. Sci. Comp. 20, 211-255, 2004.

Sweeby P (1984) High resolution schemes using flux limiters for hyperbolic conservation laws SIAM Journal on Numerical Analysis 21 995-1011.

Toro E (1999 )Riemann Solvers and Numerical ethods for Fluid Dynamics (Berlin: SpringerVerlag).

Van Albada and al (1982) Jour. Of Astron. Astrophysics.

Van leer B. ( 1974) Towards the Ultimate finite difference scheme. II. Monotonicity and conservation combined in $2^{\text {nd }}$ order scheme. Jour. Comp. Physics, 14:361-370.

Vinokur M. and Montagné J. L. (1990). Généralized flux vector splitting and Roe average for an equilibrium real gas. Journal of Comp. Physics, 89(2):276-300.

Woodward P and P. Collela P. ( 1984 ) The numerical simulation of 2D fluid flow with strong shocks. Jour. Comp. Physics; 54:115-173.

Wüthrich S, Perrel F, Sawley ML and Lafon A. (1994) Hypersonic flow in chemical non equilibrium: Comparison of thin layer NavierStokes and coupled Euler /boundary layer computations. Computers and Fluids.

Yee HC and Harten A. (1987) Implicit TVD schemes for hyperbolic conservation laws in curvilinear coordinates. AIAA Journal, 25(2):266-274.

Yee HC, (1987) Construction of Explicit and Implicit Symmetric TVD Schemes and Their Applications J. Comput. Phys. V68, pp. 151179.

Yee HC, (1989) A class of High resolution explicit and implicit Shock capturing Methods, VKI lectures, .

Yee HC, Sjögreen B, Sandham ND and Hadjadj A. (2000) Progress in the Development of a Class of Efficient Low Dissipative High Order ShockCapturing Methods, Proceedings of the Symposium in Computational Fluid Dynamics for the 21 st Century, July 15-17, Kyoto, Japan.

Yee HC, Warming RF and Harten A. ( 1985) Implicit Total Variation Diminishing TVD schemes for steady state calculations. Journal of Comp. Physics. 57(3): 327-360. 